\title{
LOS MECANISMOS DE INCLUSIÓN SOCIAL: ASPECTOS CONTROVERSIALES EN EL PROGRAMA NACIONAL DE PRODUCCIÓN DE BIODIESEL EN BRASIL
}

\author{
C. A. CASTELLANELLI \\ Universidade Federal de Santa Maria \\ castellanelli@bol.com.br
}

Artículo recibido en abril/2015 y aceptado en enero/2016

DOI: $10.15628 /$ holos.2016.2956

\section{RESUMEN}

En casi una década de funcionamiento, el Programa Nacional de Producción de Biodiesel en Brasil, hay una serie de problemas relacionados con la inclusión social de las familias campesinas. Las acciones del gobierno junto con las empresas productoras no han logrado el resultado deseado en el comienzo de la operación del programa.
No se están haciendo acciones correctivas para un verdadero trabajo de inclusión social, y por lo tanto uno de los objetivos del programa, que es la erra dicación de la pobreza y mejores condiciones de vida para las familias participantes está fallando.

PALABRAS CLAVE: Biodiesel, Inclusión Social, Programa Nacional de Producción de Biodiesel, Sello Social.

\section{THE MECHANISMS OF SOCIAL INCLUSION: CONTROVERSIAL ISSUES CONCERNING THE NATIONAL PROGRAM FOR BIODIESEL PRODUCTION IN BRAZIL}

\author{
ABSTRACT \\ In almost a decade of operation, the National Program for \\ Biodiesel Production in Brazil, has a number of problems \\ related to the social inclusion of the rural families. The \\ government actions together with the producing \\ companies have not achieved the desired result in the
}

inicial plan of the program. Corrective actions are not being made for a real social inclusion, and thus one of the important goals of the program, which is the eradication of poverty and better living conditions for the participating families is failing.

KEYWORDS: Biodiesel, Social Inclusion, National Program for Biodiesel Production, Social Seal. 


\section{INTRODUCCIÓN}

La era del petróleo puede estar llegando al fin. Algunos estudios indican que el pico de producción del petróleo está próximo de ser alcanzado. Otros más pesimistas, garantizan que la fuente ya se encuentra en el lado descendiente de la llamada "Teoría del pico de Hubbert", demostrando que más de la mitad de las reservas recuperables ya fue descubierta y el mundo pasará por un cambio que traerá nuevos actores para la escena principal de la geopolítica de la energía mundial.

Según Rifkin (2011), si la producción de petróleo llega a su pico en los próximos diez años, y si a eso se sigue la extenuación de producción global de gas natural, el resultado acarreará una serie de acontecimientos capaces de solapar en gran parte el estilo industrial de vida. En este escenario, despuntan con gran expectativa el desarrollo de tecnologías que posibilitan la utilización de fuentes alternativas de energía, como las provenientes de la biomasa, de la fuerza de las olas, la energía solar, eólica, la célula de hidrógeno, entre otras. Brasil se insiere oficialmente en esta cuestión a partir de la aprobación del marco regulador de biodiesel. El país posee una gran ventaja comparativa en relación a los países que ya producen biodiesel, que es la biodiversidad.

El futuro próximo se muestra para la posibilidad de haber grandes cambios en la fabricación y utilización de biodiesel por Brasil. La expectativa es que el país sea uno de los principales fabricantes y consumidores de biocombustibles del mundo. Hay indicios de que esto significańa la disminución de gases y del calentamiento global y consecuentemente permitiría el uso del petróleo y sus derivados para otros fines, destacando que según la visión de Capra (1996), la producción de biodiesel debería ser hecha a partir de una visión ecológica, o sea, con la producción encajada en el ambiente natural y social y así traer beneficios a la sociedad y al planeta.

El biodiesel surge no sólo como una nueva fuente de energía renovable y una oportunidad de ganancias económicas, sino también como combustible socio-ambiental, visto que este podrá proporcionar una reducción en la emisión de gases contaminantes y conforme Benedetti, Rathmann y Padula (2006), una mayor utilización de mano de obra en la cadena productiva, promoviendo así la inclusión social de los brasileños menos favorecidos.

Según el Gobierno Federal, el biodiesel permitiría el ahorro de divisas con la importación de petróleo y aceite diesel, tratándose de una ventaja estratégica al reducir la dependencia de las importaciones de petróleo. El Ministerio del Desarrollo Agrario (MDA, 2007) destacaba como principales ventajas la oportunidad para el sector agrícola, la reducción de emisión de contaminantes en comparación al diesel y la disminución del calentamiento global, con la absorción de $\mathrm{CO} 2$ durante su ciclo productivo.

Basado en las ventajas socio-ambientales atribuidas al biodiesel y principalmente en la oportunidad de incrementar la economía brasileña, el Gobierno Federal creó el Programa Nacional de Producción y Uso del Biodiesel (PNPB), un programa que busca estratégicamente el apoyo técnico a la producción y al uso de biodiesel y la inclusión de la agricultura familiar a la cadena de producción.

Un grupo de trabajo interministerial, encargado de la elaboración del Programa, evaluó que restringiendo el concepto de biomasa a los aceites vegetales del Programa, también, podría minimizar las disparidades socioeconómicas entre las regiones del país. Esa minimización se daría 
a través de la generación de empleo y recursos para agricultores familiares que proveyeran materia prima para los productores de biodiesel, y así en pro de esta meta, se construyó el Sello Combustible Social, por medio del Decreto 5.297 de diciembre de 2004.

El Sello Combustible Social es un mecanismo de modernización, fortalecimiento y dinamización de arreglos productivos, destinado a minimizar disparidades regionales, por medio de la generación de nuevos empleos rurales y rurales no agrícolas, sobre todo en el Noreste del país. Para desempeñar estas funciones la reglamentación del mercado brasileño de biodiesel, por medio de este instrumento, impone que los productores innoven sus estructuras de gobernanza interna obligándolos a adquirir su materia prima de un segmento de mercado que no tiene tradición en producirla (agricultura), bajo pena de no comercializar el biocombustible con las distribuidoras de combustibles, como estrategia para el surgimiento y consolidación de nuevos arreglos productivos que insieran a los agricultores familiares en el mercado energético.

Las plantas de producción de biodiesel con Sello Combustible Social pueden comprar directamente de los agricultores familiares o de sus cooperativas agropecuarias. Con la venta colectiva, en cantidades mayores, es posible negociar mejores precios con las empresas. Hay también ganancias con el transporte de la materia prima hasta el punto de recepción de forma colectiva y es posible comprar equipos de beneficio de los granos, como desgranador, pelador y secadores, aumentando el valor del producto y mejorando la calidad. Actualmente, las ventas de las cooperativas para las plantas de producción ya representan más de dos tercios de la venta de la agricultura familiar. La empresa de biodiesel con Sello está obligada a proveer asistencia téc nica a los proveedores y en caso se cooperativas, la tarea puede ser asumida por profesionales propios. El costo puede incluirse en el producto, o por medio de un contrato a parte.

\title{
2 EL PROGRAMA NACIONAL DE PRODUCCIÓN DE BIODIESEL Y LA INCLUSIÓN SOCIAL
}

\begin{abstract}
Además de ser un combustible limpio, que no contamina el medio ambiente, la producción industrial del biodiesel y el cultivo de materias primas ayudan a generar ingresos y trabajo para la agricultura familiar, estimulando la inclusión social, principalmente en las regiones Norte, Noreste y semiárido brasileño... La agricultura familiar de todas las regiones participa activamente en esta cadena productiva por medio de la producción y venta de sus diversas materias primas oleaginosas, como la soya, la higuerilla, el dendé, el girasol, la canola y el cacahuate. (MDA, 2012)
\end{abstract}

En mediados de 2003, en el gobierno de Luís Inácio Lula da Silva, fue creado un grupo de trabajo interministerial (GTI), coordinado por la Casa Civil de la Presidencia de la República, para realizar estudios sobre la variable de utilización de aceites vegetales para fines energéticos, con el interés de definir las bases de un programa de ámbito nacional para la producción y uso de biodiesel. En el mismo año el gobierno federal creó la Red Brasileña de Tecnología de Biodiesel (RBTB), formada por entidades de investigación distribuidas en 23 Estados brasileños, pretendiendo converger los esfuerzos de los diversos actores involucrados en la investigación, desarrollo y producción de biodiesel (Brasil, 2010). Esa articulación de esfuerzos (grupo interministerial y la red de investigación), en pro de la inclusión de biodiesel en la matriz energética 
brasileña, recibió el nombre de Programa Nacional de Producción y Uso de Biodiesel (PNPB). Así, se dieron, por tanto los primeros paros para la creación de un ambiente institucional para el mercado de Biodiesel.

La consolidación de la estrategia de crecimiento sustentable con inclusión social presupone la sustitución de este proceso asimétrico de desarrollo socioeconómico de consecuencias negativas tanto para las áreas atrasadas como también para las regiones más prósperas. Estas ya están enfrentando los problemas de aglomeración excesiva: congestionamiento de flujo, presión sobre el medio ambiente y la salud pública, incapacidad de absorber la presión migratoria, ampliando los bolsones de pobreza en todas las grandes ciudades del país. (Brasil, 2004-2007)

El desarrollo social pregonado por el PBPB se justifica por la generación de empleo e ingresos a la agricultura familiar inserida en el proceso de producción de materia prima para la producción del biodiesel, según Lima (2004), en estudios realizados por el Ministerio del Desarrollo Agrario (MDA); Ministerio de la Agricultura, Pecuaria y Abastecimiento (MAPA); Ministerio de la Integración Nacional y Ministerio de las Ciudades, muestran que para cada 01\% de sustitución del aceite diesel por el biodiesel, producido con la participación de la agricultura familiar, podrían ser generados aproximadamente cuarenta y cinco mil empleos en el campo.

De acuerdo con el mismo autor, admitiendo que para cada empleo generado en el campo se generan 3 en la ciudad, con la sustitución de diesel por el biodiesel en 1\% serían creados aproximadamente ciento ochenta mil empleos. De esta forma, con la participación de $6 \%$ de la agricultura familiar en la producción de materia prima para el biodiesel sería suficiente para general un millón de empleos.

Otro aspecto importante señalado por el autor es la comparación de la creación de puestos de trabajo entre la agricultura familiary la empresarial; mientras que el porcentaje de empleo en el campo creado por la agricultura empresarial es de 1 trabajador para cada 100 hectáreas cultivadas, en la agricultura familiar son creados 10 puestos de trabajo para cultivar esta misma área. Esto significa la importancia de priorizar la agricultura familiar en la producción de biodiesel, la oportunidad de sacar de la zona de pobreza un gran contingente de personas, principalmente del semiárido nordestino donde viven aproximadamente dos millones de familias en estas condiciones.

La inclusión social y el desarrollo regional, es especialmente vía generación de empleo e ingresos, deben ser los principios orientadores básicos de las acciones direccionadas al biodiesel, lo que implica decir que su producción y consumo deben ser promovidos de forma descentralizada y no excluyente en términos de rutas tecnológicas y materias prima utilizadas (Lima, 2004).

Para promover los cambios sociales pretendidos por el PNPB y fomentar el desarrollo regional y la participación de la agricultura familiar en el proceso productivo, el gobierno creo políticas públicas que subsidian las empresas productoras de biodiesel que adquieren materia prima oriunda de regiones menos favorecidas y producidas por la agricultura familiar. La principal herramienta utilizada para esta finalidad el Sello Combustible Social ofrecido a los productores por el MDA. 


\section{EL SELLO SOCIAL}

El Sello Combustible Social es una herramienta del PNPB creada para promover el desarrollo de la agricultura familiar y fue introducido a través del Decreto № 5.297 del 06 de diciembre de 2004 que establece las directrices del Sello y determina el MDA como agente normalizador, siendo una identificación concedida por el MDA a los productores de biodiesel que promueven la inclusión social y el desarrollo regional por medio de la generación de empleo e ingresos, donde los beneficiados son los agricultores familiares encuadrados en los criterios del Programa Nacional de Fortalecimiento de la Agricultura Familiar (PRONAF).

Según el MDA, a través del Sello Combustible Social el productor de biodiesel tiene acceso a alícuotas del Programa de Integración Social (PIS), del Programa de Formación del Patrimonio del Servidor Público (PASEP) y de la Contribución para el Financiamiento de Seguridad Social (COFINS) con coeficientes de reducción y acceso las mejores condiciones de financia miento junto al Banco Nacional de Desarrollo Económico y Social (BASA), Banco del Noreste de Brasil (BNM), Banco de Brasil S/A (BB) y otras instituciones financieras que posean condiciones especiales de financiamiento para proyectos con Sello Combustible Social. El productor de biodiesel también podrá usar el Sello para fines de promoción comercial de su empresa.

Es concedido el Sello Combustible Social al productor que atienda los criterios propuestos por el MDA, el cual exige la compra de materia prima de la agricultura familiar en un porcentual mínimo de $30 \%$ en las regiones Noreste, Sureste y Sul y el $10 \%$ en las regiones Norte y CentroOeste, pasando a ser de 15\% a partir de las producción 2010/2011. El MDA también exige que el productor firma contrato con los agricultores familiares, contrato que necesariamente debe contener:

- el plazo contractual;

- el valor de compra y criterios de reajuste del precio contratado;

- las condiciones de entrega de la materia prima;

- los salvaguardas de cada parte;

- identificación y concordancia de una representación de los agricultores que participó en las negociaciones.

- aseguren asistencia y capacitación técnica a los agricultores familiares.

Además de eso, la empresa productora de biodiesel tiene la obligación de informar al MDA los datos del contrato con los agricultores o con sus cooperativas, las adquisiciones contratadas y la asistencia técnica realizada a los productores.

Con disposición a dejar de lado los tributos federales generados en el proceso de industrialización y distribución de biodiesel en pro de la generación de empleo e ingresos en la producción primaria, producida en las regiones pobres y con mano de obra de la agricultura familiar, el gobierno espera hacer de la producción del biodiesel un proceso del desarrollo social y reductor de las diferencias regionales. 


\section{LA PROBLEMÁTICA DEL SELLO SOCIAL}

Cuando el gobierno lanzó el Programa Nacional de Producción y Uso de Biodiesel (PNPB) en 2004, los planes más ambicios os estaban en la inclusión social. Históricas desigualdades sociales se corregirían con el programa a través de participación de la agricultura familiar en la cadena productiva del biodiesel. Para eso, el Sello Combustible Social se creó priorizando determinadas regiones y plantas, como la higuerilla (mamona) y el dendé. Los mecanismos del sello creado por el gobierno en 2004, en el lanzamiento del PNPB, se fueron mostrando insuficientes a medida que el programa evolucionaba y acabaron por impedir una participación efectiva de los agricultores de bajos ingresos. Y también garantizaron que la soya, planta de gran dominio entre los latifundios, como la más utilizada por las plantas productoras y por la agricultura familiar, pues exige tecnología y grandes áreas para atender niveles interesantes de productividad. En 2008, en Ministerio de Desarrollo Agrario (MDA), admitió que el número de productores rurales en el programa no llegó a la mitad de lo esperado.

El Sello Combustible Social ganó nuevas reglas a finales de febrero de 2009 para intentar un nuevo impulso la participación de la agricultura familiar en el programa de biodiesel. Los cambios fueron tímidos, con el estímulo a la asistencia técnica y a la adquisición de oleaginosas alternativas a la soya, además de la disminución del porcentual de compra para el Noreste y SemiÁrido.

El sector productivo venía reivindicando reformas en la normativa desde 2006. La principal reclamación era con relación a la asistencia técnica de los agricultores, cuya responsabilidad fue transferida en gran parte para el fabricante de biodiesel, que por lo tanto, ha visto sus costos aumentar. Por la nueva normativa, la asistencia técnica deberá continuar en las manos de las plantas productoras.

Si por un lado las empresas productoras de biodiesel tuvieron dificultad en adquirir materia prima en las cantidades establecidas por las antiguas reglas del sello, por otro, para la agricultura familiar también es un reto atender a las necesidades de la industria. La agricultura familiar también es un estado decadente, hay muchos productores morosos y endeudados, tierras deterioradas en una fuerza de trabajo comprometida por el éxodo de los jóvenes para las ciudades. La mecanización está distante, no es interesante para el joven regresar al campo.

La falta de tecnología en los campos de higuerilla (mamona), dendé, girasol, entre otros cultivos, no se refiere solo al uso (o desuso) de máquinas, sino también al conocimiento de las mejores técnicas de plantío, desarrollo de semillas genéticamente superiores y utilización de los insumos y de los defensivos más adecuados, o sea: tecnología, pesquisa y asistencia técnica. Hoy la única oleaginosa que tiene toda su cadena productiva mapeada es la soya - y no por casualidad la producción nacional está centrada en ésta. Solamente la política de incentivo fiscal no lleva a la diversificación de la matriz. En última instancia, solo favorece a la soya como materia prima, ya que el cultivo más investigado y tecnificado es con más productividad. Proveer de materia prima las industrias de biodiesel es poco: la agricultura familiar quiere y necesita más. El agricultor es un mero proveedor de materia prima. Esto es un error en el proceso.

La agricultura familiar, para ser económicamente sustentable, necesita asistencia no solo para la producción de las oleaginosas que serán compradas por las plantas productores de biodiesel, sino también para otros culticos consorciados. La asistencia se restringe a las 
oleaginosas, no dando atención al conjunto de las actividades de la unidad de producción, lo que es insuficiente para garantizar que los agricultores tengan un buen desempeño.

En este contexto, la gestión de las políticas públicas para la producción de semillas oleaginosas por agricultores familiares para satisfacer los requisitos de los programas de biocombustibles del gobierno, el Programa Nacional de Apoyo a la Agricultura Familiar - PRONAF permanece como una política que guía el desarrollo de esta categoría productiva y tiene los servicios de la Asistencia Técnica y Extensión Rural (ATER) contratada por la Petrobras, por ejemplo, como la asistencia técnica para empresas y Extensión Rural del Estado de Minas Gerais EMATER / MG (principalmente en la ciudad de Matias Cardoso y alrededores), Cooperativa Agricultores Familiares de la Fazenda Santa Maria - COOPERSAM (Rio Pardo de Minas y sus alrededores), y la Cooperativa Grande Sertão, la garantía de un servicio con el fin de satisfacer las necesidades de la agricultura familiar de una manera consistente con la las estrategias de desarrollo del país. Otros principios igualmente importantes de ATER se refieren a la garantía de los agricultores familiares para acceder al servicio post-venta y la extensión pública, gratuita, de calidad y en cantidad suficiente, con la intencíon a su fortalecimiento, la adopción de un enfoque multidisciplinario e interdisciplinario para favorecer la práctica de la agroecología; la observación de las peculiaridades de las diferentes cadenas que cubren todas las etapas des de la producción hasta la comercialización, logística y suministro; la generación de nuevas fuentes de ingresos a través de estrategias de mejora de los mercados locales y de inserción no subordinada de la agricultura familiar en el mercado global.

Como contrapunto, según el presidente de la Unión de Trabajadores Rurales de Río Pardo de Minas, para las explotaciones familiares no hay avances positivos, pero negativos. Las explotaciones familiares ya tienen su propia ocupación, la llegada de los monocultivos, la soja, la caña de azúcar a gran escala genera puestos de trabajo, pero interrumpen la ocupación de muchos agricultores. (Informe basado en audio y señala el taller "Los biocombustibles y la Justicia Ambiental celebrada en Kochi, el 6 y 7 de mayo de 2010).

Un granjero tejió el comentario de que "gracias a Dios" que Petrobras sólo entiende algunos aspectos, porque aquellos que entienden la agricultura son los agricultores, respaldó las conversaciones anteriores que dicen los agricultores deben organizarse, ya sea en cooperativas, asociaciones, por último, lo que sea necesario para fortalecerse ante el poder de las grandes empresas, para que puedan obtener las mejores condiciones de negociación en el mercado. Otros de los agricultores familiares reportaron su lucha en un conflicto por el uso del suelo: Lo que lo que más necesitados es una protección de la tierra, la tierra y el agua.

En respecto de las cuestiones financieras, un agricultor de Montezuma - MG, informó su disgusto por préstamo bancario y presenta la instancia de crédito a disposición de los agricultores locales para plantar ricino para la empresa PBBio, lo que resulta en mora por no cumplir con el compromiso asumido por el entrenador COOPERSAM en nombre de PBBio. Además de eso hay una cierta resistencia por parte de los agricultores en la aceptación de nuevas técnicas de plantío. Nadie hace experimentación con aquello que va a ser el sustento de la familia durante dos años con el riesgo de perder todo.

El Ipea (2013), realizó un análisis del PNPB, el cual muestra que la meta inclusión social estipulada por el programa no fue alcanzada. El uso de materias primas que exigen uso intensivo de mano de obra como el dendé y la higuerilla (mamona), fue prácticamente insignificante. La soya 
reinó absolutamente en el mercado, e aun cuando el grano fue adquirido de agricultores familiares, vino de propiedades que ya explotaban este cultivo y serian incluidas en la cadena productiva de biodiesel de cualquier forma.

En regiones con nivel educacional elevado, una planta de biodiesel necesita invertir mucho menos para hacer que el pequeño agricultor produzca la materia prima deseada. No importa se va a ser higuerilla (mamona), soya o canola. La planta en la región Noreste necesita investigar más en asistencia técnica, enseñarles las ventajas de una cooperativa, proveerles maquinaria y varias cosas que muchas veces no son necesarias en la región Sur.

Los diferentes periodos entre los productores de las regiones, aliados al mercado de soya ya establecido, hicieron que el programa de incentivo a la agricultura familiar incentivase a los agricultores que ya tenían a quien vender su producción. Tal vez el mayor error del Programa Nacional de Producción y Uso de Biodiesel fue haber tratado todas las oleaginosas de la misma manera, con los mismos estímulos. Esto llevó a las plantas productoras a optar por lo que ya estaba listo. Si oleaginosas alternativas ofrecieran un retorno mayor al productor de biodiesel, es posible que, transcurridos esos casi cuatro años de obligatoriedad en el uso de biodiesel, la soya no representara más del $80 \%$ de materia prima usada y la región Sul no se hubiera vuelto la región propaganda del MDA a la hora de presentar resultados.

El estudio muestra que aunque el Sello Combustible Social sea importante dentro del mecanismo del PNPB, no ha cumplido su papel como fuente de estímulo para el pequeño productor rural. Ha significado un mecanismo de incentivo para la industria, pero no de soporte de los objetivos de inclusión del PNPB. Esto porque, en vez de alcanzar al pequeño agricultor, que era el objetivo inicial, el sello se convierte en un facilitador que permite acceso a los incentivos fiscales para las industrias, además de facilitar su acceso al mercado.

\section{POSIBLES OBSTÁCULOS A LA PRIORIZACIÓN DE LA INCLUSIÓN SOCIAL}

El impacto del biodiesel en la facturación de tradicionales empresas brasileñas de la soya, como Granol, Caramuri y ADM, fue grande. No es coincidencia que justamente esas empresas encabecen la lista delas que más invierten en el mercado de biodiesel. Analistas piensan que el dominio de las aplastadoras tiende a aumentar con el rumbo que el PNPB está tomando, ya que las reglas no estimulan otros cultivos. La decisión de cuál oleaginosa va a ser utilizada para hacer biodiesel acaba siendo mercadológica.

Los acontecimientos que condujeron la producción del biodiesel al modelo actual revelan una gran verdad: para las industrias, la producción de biodiesel es una actividad comercial y sigue las leyes de mercado, donde la competitividad de un producto padrón se da por menor precio y el menor precio es posible a través de la compra de materia prima barata y menor costo de logística.

Es importante recordar que por medio del PNPB, el Gobierno Federal organizó la cadena productiva, definió las líneas de financiamiento, estructuró la base tecnológica y editó el marco regulatorio del nuevo combustible.

Bajo la óptica de Almeida (2010),esta intervención gubernamental directa en la economía de mercado no es extraña frente a los moldes capitalistas, que expresan que la economía debe auto regularse por el mercado, excluyendo el estado como elemento regulador para estimar mayores lucros y en otros momentos cuando son necesarias inversiones de riesgo y a fondo 
perdido, suplican el amparo estatal, para garantizar precio mínimo, mercado consumidor, condiciones subsidiadas de producción, haciendo así que las arcas públicas se aventuren en los costos y riesgos del emprendimiento y los lucros puedan ser privatizados.

Se puede citar otro factor posiblemente actuante seria la Responsabilidad Social Empresarial (RSE), o la falta de la misma, que viene ocupando lugar de destaque en la literatura de negocios en Brasil. En los últimos treinta años el debate acerca de ese tema pasó de una posición marginal para transformarse "omnipresente, sea en los ambientes corporativos o en los organismos internacionales, y tanto entre movimientos de la sociedad civil como en los pasillos académicos"(Kreitlon, 2004, p. 2).

Se suma a eso, el hecho de que las empresas han ampliado sus ramos de actuación y deben estar más atentas no solo al cumplimiento de sus responsabilidades económicas y legales, pero deben considerar también las implicaciones de sus actividades (Veloso, 2005): prácticas, políticas y comportamientos esperados ( en el sentido positivo) por miembros de la sociedad, a pesar de no estar codificados en leyes (...) una serie de normas, padrones o expectativas de comportamiento para atender aquello que los diversos públicos (stakeholders) con los cuales la empresa se relaciona consideran legítimo, correcto, justo o de acuerdo con sus derechos morales o expectativas (Veloso, 2005, p. 5).

Sin embargo, no hay solamente una única definición para el concepto de RSE, de forma que no existe un consenso sobre sus significados y límites, y el concepto presenta diferentes desdoblamientos y usos por las empresas como un todo (Carrol, 1999; Ventura, 2005). Entre las diferentes perspectivas sobre el tema, verificamos que este evolucionó a lo largo de los años de una visión conservadora en que la empresa socialmente responsable es aquella que responde solo a las expectativas de sus accionistas, hasta una visión la cual indica que la empresa debe también enfrentar el desarrollo político y legal a favor del interés público, pues debe estar atenta también a las expectativas de sus stakeholders actuales y futuros (Tomei, 1984; Ashley, 2005).

Según Ashley (2005), la visión conservadora puede ser ilustrada a través delas ideas de Friedman y Leavitt. El primero afirma que la responsabilidad social es un comportamiento antimaximización de lucros, pues beneficia otros agentes que no son los accionistas de la empresa. De esta forma, "la dirección corporativa, como agente de los accionistas, no tiene el derecho de hacer nada que no atienda al objetivo de maximización de los lucros, mantenidos los límites de la ley".(Ashley, 2005, p. 48).A este argumento puede agregarse la función institucional propuesta por Leavitt, que indica las actividades de responsabilidad social construidas como una sobretasa al lucro de los accionistas, una vez que dentro de la sociedad existen otros agentes además de la corrupción - como gobierno, iglesias, sindicatos y organizaciones sin fines de lucro- aptos para actuar en actividades de responsabilidad social. Así, la empresa no debe asumir otras responsabilidades que no a la maximización de la utilidad de los detentores de sus acciones. Esos diferentes argumentos forman parte de contextos históricos y sociales distintos y llevan a la percepción de que el papel de las empresas viene siendo repensado y reformulado (Faria e Sauerbronn, 2005).

Los movimientos sociales que reivindicaron global o localmente un nuevo tipo de sociedad, vislumbran un futuro diferente de aquel del horizonte empresarial. Sadler (2004), también indica la posibilidad de haber relación entre el crecimiento de la campaña de responsabilidad social de las empresas en todo el mundo y el concomitante aumento de movimientos sociales en reclamo 
también a nivel global. El autor se cuestiona: “¿Hasta qué punto la responsabilidad social empresarial no es simplemente un medio de neutralizar fuerzas sociales opuestas?"

El futuro vislumbrado por las grandes empresas establece en el presente sumedida. Gomes (2002, p.22) cree que "el discurso relatado por empresas o instituciones que se auto-nombran, o son reconocidas por algún órgano, responsables sociales, están, de hecho, colaborando más para la manutención del status quo que realmente para estimular la solidaridad.

Como solución a la protección del medio ambiente, hay acuerdos privados, cuyo objetivo es ayudar y dar un norte más sostenible a la producción, como las llamadas mesas redondas, donde los actores de diferente naturaleza negocian las reglas para ser adoptado por los organismos reguladores - de soja, aceite de palma, biocombustibles, etc. En función de la evolución de la negociación, la institución podrá expedir un certificado / sello de certificación de la conformidad de los países adherentes como una manera de aumentar el nivel de información sobre la cadena de suministro, y ayudar a los actores individualmente a tomar la mejor decisión. Entre ellos, podemos mencionar la RTRS, Greenergy, ISCC, RSB, Soy Plus, entre otros.

La planta de biodiesel de Cargill, por ejemplo tiene los certificados de ISCC y 2BSvs que confirman que los biocombustibles producidos por la empresa obedecen los requisitos ambientales de la Unión Europea (UE). Instalado en Três Lagoas (MS), la planta es la sexta más grande de Brasil, con una capacidad instalada de hasta 252 millones de litros por año. Para garantizar la sostenibilidad en toda la cadena, la Cargill incluye en el ámbito de las certificaciones productores que forman parte del Programa de Agricultura Familiar en Mato Grosso do Sul.

EI ISCC certifica que el biodiesel producido cumple con los estándares sociales y ambientales establecidos por la Directiva Europea de Energías Renovables, que está a años luz por delante de lo Sello Social propuesto. Acciones como esta, sin embargo, están aislados en el contexto de PNPB.

\section{CONSIDERACIONES FINALES}

Todo y cualquier programa energético contienen en su esencia, una preocupación con el bienestar (social y/o económico) de aquellos que son su público objetivo. Sea un programa destinado a la conservación de energía o a la expansión de su oferta, su meta será siempre la mejoría de la calidad de vida de aquellos que de él se beneficiarán.

El Sello Combustible Social es un mecanismo de modernización, fortalecimiento y dinamización de arreglos productivos, destinado a minimizar disparidades regionales, por medio de la generación de nuevos empleos rurales y rurales no agrícolas, sobre todo en el Noreste del país. Para desempeñar estas funciones la reglamentación del mercado brasileño de biodiesel, por medio de este instrumento, impone que los productores innoven sus estructuras de gobernanza interna obligándolos a adquirir su materia prima de un segmento de mercado que no tiene tradición en producirla (agricultura), bajo pena de no comercializar el biocombustible con las distribuidoras de combustibles, como estrategia para el surgimiento y consolidación de nuevos arreglos productivos que insieran a los agricultores familiares en el mercado energético.

EI PNPB es un programa de política pública todavía muy reciente y con un horizonte temporal muy largo ensu frente, donde pueden ser identificadas y corregidas algunas fallas. Sin embargo, la ausencia de líneas de crédito, la baja participación de la agricultura familiar, así como, 
la alta utilización de soya en la producción de biodiesel pone en riesgo el carácter social y ambiental do PNPB, así como del biodiesel, como elemento propulsor de desarrollo para las áreas rurales. Por lo tanto, se puede concluir que los principales beneficios anhelados por el Programa Nacional de Producción y Uso de Biodiesel (PNPB), llevan a un escenario de no sustentabilidad. Esa información es hecha a partir de la observación de varias anomalías sociales que circundan el programa como problemas en el uso del suelo: monocultivo, deforestación, pérdidas biológicas, uso intensivo de fertilizantes y defensivos agrícolas, dominio de la cadena por el agro-negocio, centralización en materias primas dominantes, poca utilización de insumos residuales y materias primas diversificadas, baja generación de empleo y ganancias; y baja participación y org anización social.

El análisis de los cambios ocurridos en el PNPB en una década de operación evidencia un distanciamiento entre los objetivos iniciales. La pretensión inicial de utilizar el PNPB para disminuir las desigualdades regionales, a través de la localización planeada de las plantas industriales y la producción de materia prima para el biodiesel en la regiones menos favorecidas económicamente, fue visiblemente abandonada a medida que la producción actual de biodiesel se concentra en las regiones Centro-Oeste, Sureste y Sur que son las regiones más desarrolladas económicamente.

En suma, uno de los objetivos del programa todavía no fue alcanzado. La inclusión de la agricultura es el gran obstáculo que estuvo presente en todo el trayecto. Para superarlo, es necesaria una gran reformulación. Con las actuales normas del Selo Combustible Social, el gobierno transfirió a las plantas de producción toda la responsabilidad por el entrenamiento y fomento de los pequeños agricultores. Como no fue hecha ninguna distinción entre los que ya produjeron y los que no producirán, las plantas de producción optaron por el camino más barato, como se espera de cualquier empresa. Así, se priorizaron los pequeños productores de soya, que no necesitaban de asistencia técnica o transferencia de tecnologías. Fue utilizada una cadena de producción consolidada, con pequeños agricultores que ya producían. O sea, no hubo inclusión familiar, solo un cambio de compradores. Hace décadas, gobierno tras gobierno, inúmeros programas para resolver la pobreza en el campo se crearon. Hubo progresos, pero el problema continua.

\section{REFERENCIAS}

1. ALMEIDA, J.P. Biodiesel o "óleo filosofal"Desafios para a educação ambiental no caldeirão do Desenvolvimento Sustentável. Biblioteca Virtual de Ciências Humanas do Centro Edelstein de Pesquisas Sociais - www.bvce.org- Rio de Janeiro, 2010.

2. ASHLEY, P. A. (Coord.). Ética e Responsabilidade Social nos Negócios. 2. ed. São Paulo: Saraiva, 2005.

3. ARROYO, M.'"Movimentos Sociais e o Conhecimento: uma relação tensa". In: II Seminário Nacional MST e a Pesquisa. Cadernos do Iterra, Veranópolis RS. Ano VII, 2007.

4. BENEDETTI, O. Uma proposta de modelo para avaliar a viabilidade do Biodiesel no Brasil. Teoria e Evidência Econômica. 14(ed. Especial), 81-107, 2006.

5. BRASIL - Plano Plurianual (2004-2007). Participação e inclusão social. Governo Federal. Disponível em: < http://www.planobrasil.gov.br/texto_base.asp?cod=5> Acesso em: 01Fev. 2015.

6. BRASIL. Programa Nacional de Produção e Uso de Biodiesel. Disponível em: 
www.biodiesel.gov.br. Acesso em 02 Fev. 2015, 2010.

7. BRASIL. Lei № 11.097, de janeiro 2005. Dispõe sobre a introdução do biodiesel na matrizenergética brasileira; altera as Leis nos 9.478, de 6 de agosto de 1997, 9.847, de 26 de outubrode 1999 e 10.636, de 30 de dezembro de 2002; e dá outras providências.

8. BRASIL. SECRETARIA DA RECEITA FEDERAL. Decreto n.5.297, de 6 de dezembro de 2004. Dispõe sobre os coeficientes de redução das alíquotas da Contribuição para o PIS/PASEP e da COFINS incidentes na produção e na comercialização de biodiesel, sobre os termos e as condições para a utilização das alíquotas diferenciadas, e dá outras providências. Diário Oficial da União, 07 dez, 2010.

9. BRASIL. MINISTÉRIO DE DESENVOLVIMENTO AGRARIO-MDA. 2009. Instrução Normativa n.1 de 19 de fevereiro de 2009. Dispõe sobre os critérios e procedimentos relativos a concessão, manutenção e uso do selo combustível Social. Diário oficial da União, 25 de fevereiro de 2009, 2009.

10. CAPRA, F. The web of life: a new scientific understanding of living systems. New York, Anchor, 1996.

11. CARROL. A. "Corporate social responsibility: evolution of a definitional construct. Business and Society", v. 38, p. 268-295, 1999.

12. FARIA, A.; SAUERBRONN, F. F. Em Defesa de uma Abordagem menos Estratégica para a Responsabilidade Social. In: Anais do II 3Es - Encontro de Estudos em Estratégia. Rio de Janeiro: ANPAD, 2005.

13. FAURE, E. Appredre a être. Paris: Fayard and UNESCO, 1972; Versiónespañola: PAREDES DE CASTRO, Carmen. Aprender a ser. La educación del futuro.ed. Madrid: Alianza Editorial, 1973.

14. GOMES, A. "Responsabilidade social, ação social e balanço social. Novos patamaresda ciência social ou mais um neologismo semântico?" in: Revis ta da ESPM. Ano 8, v.9, ed.4Julho/Agosto. p.20-31, 2002.

15. IPEA. Instituto de Pesquisa Econômica e Aplicada - www.ipea.gov.br , Rio de Janeiro, 2013.

16. KREITLON, M. P. A. "Ética nas Relações entre Empresas e Sociedade: Fundamentos Teóricos da Responsabilidade Social Empresarial". In: Anais do XXVIII ENANPAD.Paraná: Curitiba, 2004

17. LIMA, P. C. R. O biodiesel e a inclusão social. Consultoria legislativa, Brasília, <http://bd.camara.gov.br/bd/bitstream/handle/bdcamara/1142/biodiesel_inclusao_lima.pdf ?sequence=1> Acesso em: 17 Jan. 2015, 2004.

18. MINISTÉRIO DO DESENVOLVIMENTO AGRÁRIO, MDA. Disponível em:<www.mda.gov.br $>$. Acesso em 03 mar. 2015.

19. POLETTI, R. Constituições Brasileiras, 1934. Brasília: Senado Federal e Ministério da Ciência e Tecnologia, Centro de Estudos Etnográficos, 2001.

20. RIFKIN. J. La Tercera Revolucion Industrial. Editor: PAIDOS. Espanã, 2011.

21. TOMEI, P.A. "Responsabilidade social de empresas: análise qualitativa da opinião do empresariado nacional". Revista de Administração de Empresas. v. 23 n. 4. pp.189-202, 1984.

22. VELOSO, L. H. M. "Ética, valores e cultura: especificidades do conceito de responsabilidade social corporativa". In: ASHLEY, Patricia (Coord.). Ética e Res ponsabilidade Social nos Negócios. 2. ed. São Paulo: Saraiva, 2005. 
23. VENTURA, E. C. F. Dinâmica de Institucionalização de Práticas Sociais: Estudo da Responsabilidade Social no Campo das Organizações Bancárias. Tese (Doutorado em Administração). Escola Brasileira de Administração Pública e de Empresas. Fundação Getúlio Vargas, 2005. 